\title{
Physical and Practical Challenges in Analytical Electron Tomography of Aluminum Alloys
}

\author{
A. Orthacker ${ }^{1,2}$, G. Haberfehlner ${ }^{1}$, J. Tändl $^{3}$, M.C. Poletti ${ }^{3}$, L. J. Allen ${ }^{4}$ and G. Kothleitner ${ }^{1,2}$ \\ 1. Graz Centre for Electron Microscopy, Graz, Austria \\ 2. Institute for Electron Microscopy and Nanoanalysis, Graz University of Technology, Graz, Austria \\ 3. Institute of Materials Science and Welding, Graz University of Technology, Graz, Austria \\ 4. School of Physics, University of Melbourne, Parkville, Victoria 3010, Australia
}

For a thorough understanding of a material, investigations at the nanoscale are often essential. Analytical techniques like electron energy loss spectroscopy (EELS) and energy dispersive X-ray spectroscopy (EDXS) in probe corrected scanning transmission electron microscopy (STEM) can reveal important chemical information necessary for the development and improvement of high-tech materials. The integrative character of the signal acquired through transmission, however, might hide important structural details of the material, relevant for its properties. Those details can be revealed through electron tomography, where the data is acquired at different tilt angles and, after alignment, reconstructed to form a full $3 \mathrm{D}$ model of the material under investigation. The combination of both techniques, analytical STEM and tomography, gives full insight into structure and composition of a material [1]. This novel technique is not yet well established and various experimental challenges remain, especially if a quantitative analysis of the material is the ultimate goal.

From previous investigations at the atomic scale it is well known that channeling effects have a great influence, not only on STEM image intensities, but also on the analytical signals detected [2]. The subject of this investigation are bulk metallic samples. Even if the tomographic reconstructions of the material of interest are not carried out at atomic resolution, but rather at the nanoscale, the question of the influence of channeling on the detected intensities remains.

In this study an aluminum alloy containing scandium $(\mathrm{Sc})$ and zirconium $(\mathrm{Zr})$ rich nano-precipitates was investigated at different stages of ageing. High resolution STEM and analytical EELS and EDX tomographic investigations were carried out. For the latter, problems such as sample damage, noisy spectra and the difficult detection of very low $\mathrm{Zr}$ concentrations were successfully tackled. The resulting 3D elemental reconstructions deliver otherwise inaccessible information on the sample's chemistry and structure.

To approach quantitative analytical tomography the influence of channeling on the detected signal intensities needs to be understood, to make sure its effect on elemental quantification results is either incorporated in the analysis or the experimental conditions are chosen to minimize such effects. Therefore a comparative study is performed on a rod shaped sample with an off-axis nano - precipitate. Reconstructions from different sets of tilt series performed using different experimental conditions such as tilt angles, convergence angles and defocus values are conducted and compared to investigate the reproducibility of analytical results under different channeling conditions.

[1] G. Haberfehlner et al, Nanoscale 6 (2014), p. 14563.

[2] G. Kothleitner et al, PRL 112, (2014), p. 85501 
[3] The authors thank the Austrian Cooperative Research Facility, the European Union (7th Framework Programme: ESTEEM2) and the Austrian Research Promotion Agency FFG (TAKE OFF project 839002) for funding.

[4] Special thanks go to Prof. Matthew Weyland and Dr. Scott Findlay (Monash University, Clayton, Victoria 3800, Australia) for their support regarding quantitative ADF-STEM imaging and simulations.
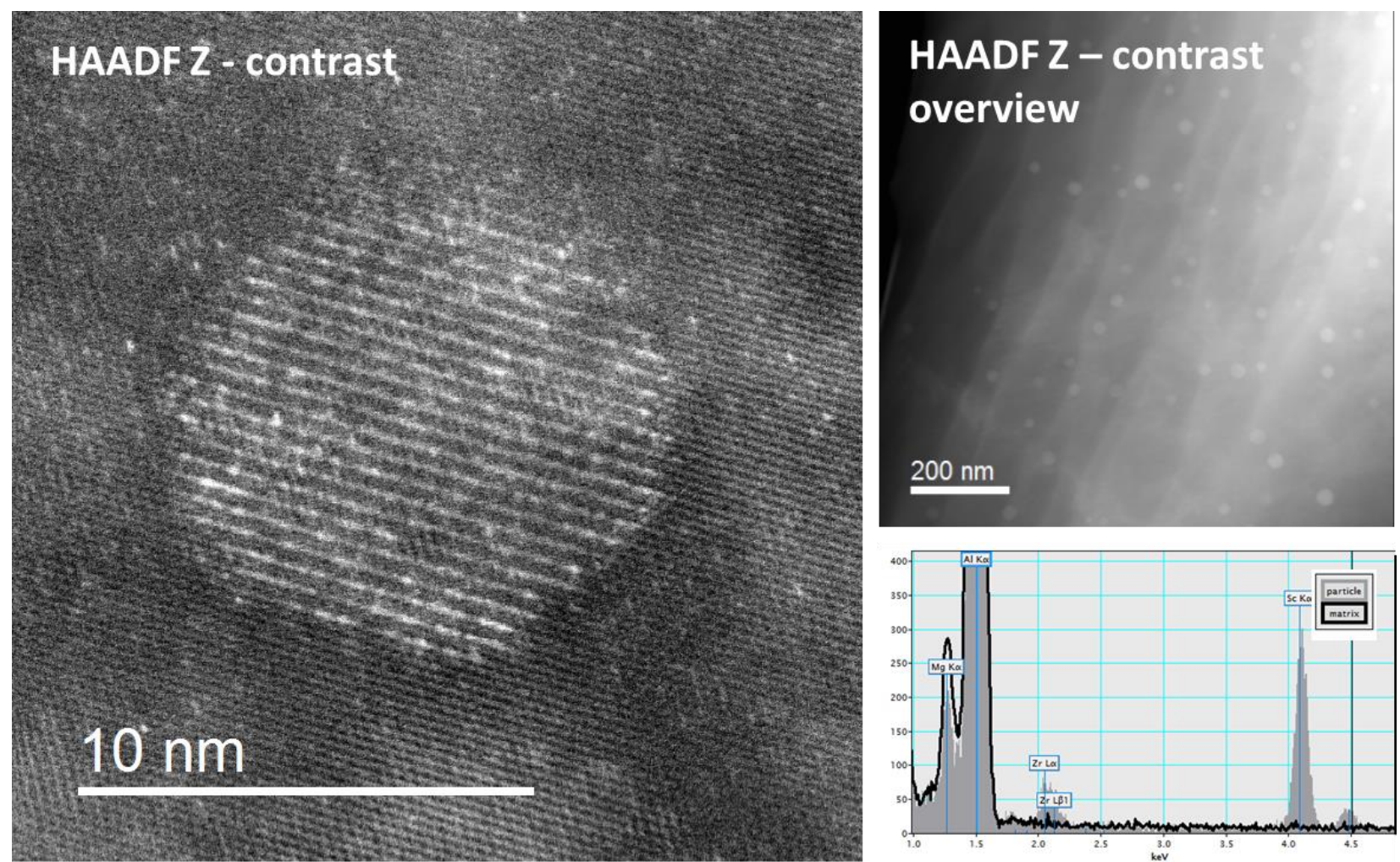

Figure 1. Left and right top: STEM HAADF images of the aluminum alloy and nano-precipitates. Bottom right: EDX spectra from a precipitate (grey) and the aluminum matrix (black). Scandium and zirconium peaks suggest that those two elements are found at the sites of the precipitates.
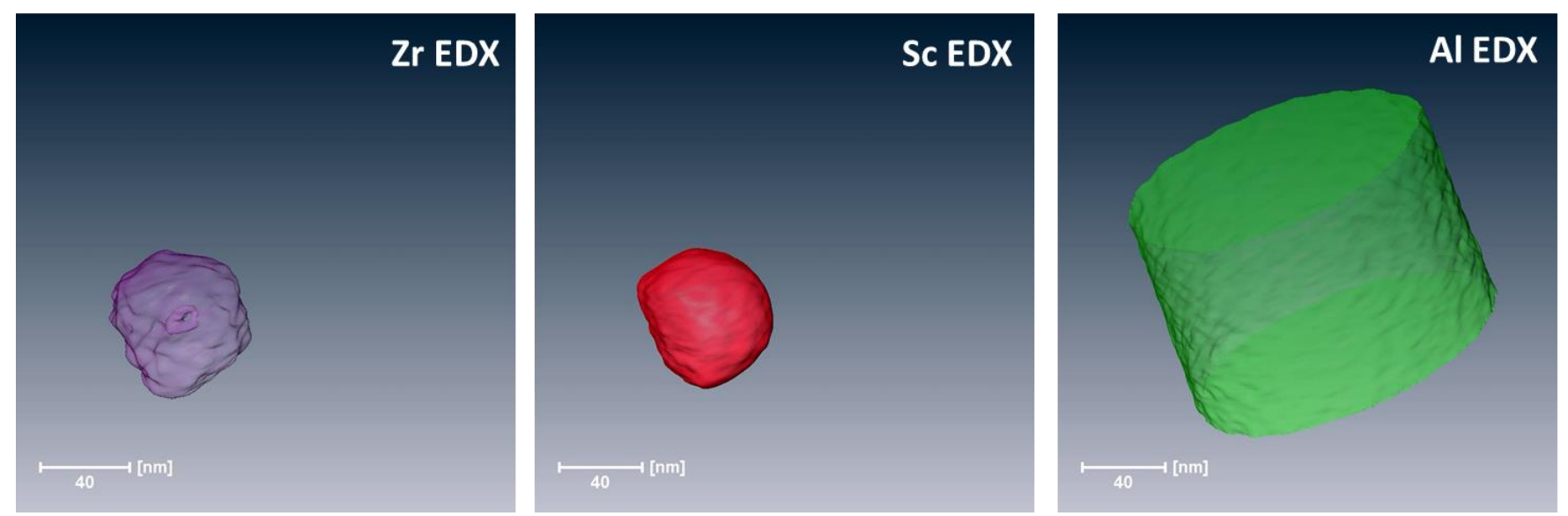

Figure 2. Segmented volumes from reconstructions of EDX signals of an aluminum alloy with nanoprecipitates after ageing for $72 \mathrm{~h}$ at $500^{\circ} \mathrm{C}$. The reconstructions were performed using a total variation minimization algorithm after processing X-ray intensities, which are subject to channeling effects. 